\title{
A BCS-Based Biowaiver Approach Using Biphasic Dissolution Test
}

\author{
Daniela Amaral Silva, ${ }^{1,2}$, Katherine J. Curo Melo ${ }^{1,2}$, Neal M. Davies ${ }^{1}$, Nadia Bou-Chacra², Humberto G. \\ Ferraz ${ }^{2}$, and Raimar Löbenberg ${ }^{1, *}$ \\ ${ }^{1}$ Faculty of Pharmacy \& Pharmaceutical Sciences, University of Alberta, Edmonton, AB, Canada. \\ ${ }^{2}$ Faculty of Pharmaceutical Sciences, University of Sao Paulo, Sao Paulo, Brazil.
}

e-mail: raimar@ualberta.ca

\begin{abstract}
Biowaivers based on the Biopharmaceutics Classification System (BCS) class can be used to establish therapeutic equivalence based on dissolution tests, which can be used as a surrogate to determine if two pharmaceutical equivalent products are interchangeable (i.e., bioequivalent). The objective of the present study was to use a biphasic dissolution system to replicate a study in which the authors followed compendial methods to examine the in vitro performance of widely used drug products. Furthermore, since biphasic dissolution studies are mainly applied to poorly soluble drugs, the present study was conducted to broaden its application to highly soluble drugs. The approach taken in this manuscript was to have scientific insight and mechanistic understanding rather than a strict regulatory application. In the initial study, none of the tested metronidazole products were (in vitro) equivalent to the comparator pharmaceutical product (CPP) or to other manufacturers. We hypothesized that whereas in vitro equivalence was not achieved in compendial methods, the partitioning profile to the organic phase in the biphasic system could signal in vitro equivalence between the tested drug products and CPP. In the aqueous phase of the biphasic system, the tested metronidazole products followed a similar pattern as obtained in the compendial buffer; however, this was not the case for the organic phase partition profiles. All the tested products had a good correlation to the CPP, which could indicate in vitro equivalence between these products. This could potentially allow for a biowaiver application. Hence, the application of biphasic dissolution to highly soluble drugs might be beneficial to estimate the product's in vivo behavior. Further clinical studies would be needed to confirm these findings to guarantee their interchangeability.
\end{abstract}

KEYWORDS: Biowaiver, in vitro equivalence, BCS, biphasic dissolution, metronidazole, dissolution

\section{INTRODUCTION}

nderstanding of the physicochemical properties of drugs through the establishment of the Biopharmaceutics Classification system (BCS) has made possible a risk-based in vitro assessment of bioequivalence for oral drug products (1). Biowaivers based on BCS class can be used to establish therapeutic equivalence. $\mathrm{A}$ biowaiver means that in vivo bioavailability and/or bioequivalence (BA/BE) studies may be waived based on in vitro dissolution testing $(2,3)$. Here, dissolution tests are used as a surrogate to determine if two pharmaceutical equivalent products are interchangeable (i.e., bioequivalent) instead of conducting expensive and time-consuming in vivo BE studies. Thus, the BCS-based biowaiver approach is intended to reduce in vivo $\mathrm{BE}$ studies and emphasizes the importance of in vitro testing for predicting in vivo performance.

In 2000, the United States Food and Drug Administration (FDA) the drafted the guidance document for industry, Waiver of In Vivo Bioavailability and Bioequivalence Studies for Immediate-Release Solid Oral Dosage Forms Based on a Biopharmaceutics Classification System (4). The European Medicines Agency (EMA) and World Health Organization (WHO) followed the FDA approach, implementing their own guidance documents in 2002 and 2006, respectively $(5,6)$. These first guidance documents differed from each other; for example, the FDA and EMA only allowed BCS-based biowaiver for BCS 
class I drugs, whereas the WHO guidance also included BCS class III and IIA (7). However, class IIA was removed from the WHO guidance in 2015. Also, the different guidance documents define "highly soluble" differently by using either the highest available strength or the highest single therapeutic dose (8). This can cause the same drug product to be classified differently in different regions of the world. Global harmonization was recommended (8).

In attempt to harmonize these various guidelines, the $\mathrm{ICH}$ published the ICH M9 Guideline on Biopharmaceutics Classification System-based Biowaivers for consultation in 2018, which has recently reached step 5 (implementation) of the ICH process (9-12). This guideline is applicable to immediate-release solid oral dosage forms or suspensions containing BCS class I or III drugs, i.e., drug products with highly soluble drug substance(s).

In 2012, Löbenberg et al. conducted a study to examine the in vitro performance of three widely used drug products marketed in different countries of the Americas metronidazole, zidovudine, and amoxicillin (all classified as BCS class I and WHO list of essential medicines) (13). The generic products in the Americas were compared to the US comparator pharmaceutical product (CPP) and to each other to determine if they met in vitro bioequivalence criteria. The authors hypothesized that the different drug products would meet the criteria due to their BCS class. However, none of the tested metronidazole products were in vitro equivalent to the CPP or to other manufacturers. Thus, since the in vitro studies did not signal that bioequivalence criteria would be met, further clinical studies would be needed to confirm their interchangeability.

The objective of the present study was to replicate Löbenberg et al.'s study using biphasic dissolution testing and metronidazole as the model drug. This is an innovative and more physiologically relevant in vitro approach than compendial methods that has been developed to effectively predict in vivo performance of drug products (14-23). The system consists of immiscible aqueous and organic phases in which the drug dissolves in the aqueous layer and partitions into the organic phase, thus maintaining sink conditions. The organic layer mimics the gastrointestinal (GI) membrane, and the dissolutionpartition process between the two phases resembles the in vivo drug dissolution and absorption process (14). Hence, the approach taken in the present study was to have scientific insight and mechanistic understanding rather than a strict regulatory application.
We hypothesized that whereas in vitro bioequivalence was not achieved in compendial methods, the partitioning profile to the organic phase in the biphasic system might signal in vitro equivalence between the tested drug products and CPP (13).

\section{METHODS}

\section{Materials}

The following commercial metronidazole tablets were purchased.

- The CPP was flagyl $250 \mathrm{mg}$ (Pfizer, USA, lot \#C071094), labeled "Flagyl-USP" [Note: Flagyl 500 mg Pfizer, Lot \#C061228, was tested with the $250 \mathrm{mg}$ lot and found similar in dissolution rate and was not used because insufficient units were available.]

- $\quad$ Flagyl 500 mg (Sanofi Aventis, Mexico, lot \#888575), labeled "Flagyl-Mexico"

- Flagenase 500 mg (Laboratorios Liomont, Mexico, lot \#7009)

- Colpofilin 500 mg (Laboratorio Lazar, Argentina)

- Metral 500 mg (Laboratorio Pablo, Cassara, lot \#77)

Metronidazole drug powder (USP grade) was obtained from Medisca (Quebec, Canada). Acetonitrile HPLC grade and 1-octanol $99 \%$ were purchased from Acros Organics (NJ, USA). The buffer solutions were prepared with purified water (Elgastat Maxima UF and an Elgastat Option 3B water purifier, ELGA Laboratories Ltd., Mississauga, ON, Canada).

The excipient composition of each drug product as listed in the package insert is as follows.

- Flagyl-USP: cellulose, Fd \& C blue, hydroxypropyl cellulose, hypromellose, PEG, stearic acid, titanium dioxide

- Flagyl-Mexico: calcium dihydrate phosphate, corn starch, magnesium stearate, povidone, hypromellose, macrogol

- Flagenase: not available

- Colpofilin: lactose, MCC, DOSSNa, povidone, crosscarmelose sodium, talcum, mg-stearate

- Metral: starch glycolate, microcrystalline cellulose, croscarmellose sodium, magnesium stearate, povidone 


\section{Analytical Quantitation}

The metronidazole content in the tablets was evaluated using high-performance liquid chromatography (HPLC) (Shimadzu LC-10AS, Tokyo, Japan) and diode array detector (Shimadzu SPD-M10AVP) UV-Vis detection at 254 $\mathrm{nm}$ using a $5-\mu \mathrm{m}(25 \mathrm{~cm} \times 4.0 \mathrm{~mm}$ ) column (LiChrospher 60 RP-Select B, Merck-Darmstadt, Germany). The mobile phase consisted of acetonitrile and water $(34: 66, \mathrm{v} / \mathrm{v})$, filtered and degassed. The flow rate used was $1.0 \mathrm{~mL} /$ min, the injection volume was $20 \mu \mathrm{L}$, and the retention time was $6.83 \mathrm{~min}$. A standard solution was prepared from an accurately weighed quantity of the reference chemical substance, using the methanol as diluent to obtain a solution of $1.00 \mathrm{mg} / \mathrm{mL}$.

\section{Disintegration Test}

The disintegration test was performed according to USP general chapter <701> (24). Disintegration time was measured in a disintegration tester (Eureka, Germany) using $900 \mathrm{~mL}$ of phosphate buffer $\mathrm{pH} 6.8$ at $37 \pm 2{ }^{\circ} \mathrm{C}$ as medium. Six tablets of each drug product were tested. Standard USP disks were used.

\section{Dissolution Tests}

All dissolution tests were performed in triplicate using a USP apparatus II (ERWEKA GmbH, Germany) with a 75 rpm rotation speed at $37^{\circ} \mathrm{C}$. All buffer media were filtered by vacuum and degassed in an ultrasonic bath.

The commercial metronidazole tablets were tested in compendial simulated intestinal fluid (SIF) $(50 \mathrm{mM}$ phosphate buffer at $\mathrm{pH} 6.8,900 \mathrm{~mL}$ ) without enzyme and in physiological buffer capacity $(5 \mathrm{mM}$ phosphate buffer at pH 6.8, $900 \mathrm{~mL}$ ). The tablets were also tested in a biphasic dissolution system in which the aqueous layer was composed of $200 \mathrm{~mL}$ of $5 \mathrm{mM}$ phosphate buffer (pH 6.8) with $100 \mathrm{~mL}$ of n-octanol on top. A mini-paddle (Sotax AG, Switzerland) was mounted on the regular compendial paddle to obtain sufficient hydrodynamics in both phases. The volume of $200 \mathrm{~mL}$ was chosen in an attempt to better approximate that of the intestinal fluids (77 $\pm 15 \mathrm{~mL}$ ) while still being experimentally feasible (25).

For both compendial and biphasic dissolution tests, samples from the aqueous phase and the organic phase were collected at specific time points $(5,10,15,20,30$, $45,60 \mathrm{~min})$.

\section{Data Analysis}

The Microsoft Excel add-in DDSolver was used to compare the dissolution profiles using similarity factor $\left(f_{2}\right)$ analysis, which measures the closeness between two profiles. According to the FDA criteria, $f_{2}$ values between 50 and 100 indicate acceptable similarity between two dissolution profiles.

The active pharmaceutical ingredient (API) must be released from the formulation and dissolve in the aqueous medium in order to partition to the organic phase. Hence, formulation disintegration is of crucial importance for drug release, especially for immediate-release tablets. With this in mind, the amount portioned into the organic phase of the CPP was correlated to the amount portioned of each one of the generic products after disintegration. The CPP (Flagyl-USP) had a disintegration time of 5:19, hence the percent partitioned from $10 \mathrm{~min}$ on (next data point after 5:19) was considered. The same rationale was used for the other products: Flagyl-Mex: 20 min on; Colpofilin: 5 min on; Flagenase and Metral: 15 min on. Table 1 shows the amount partitioned for each drug product used in the correlation. The correlation was done by linear regression.

Table 1. Amount Partitioned (Q\%) into the Organic Phase for Each Drug Product After Disintegration Time

\begin{tabular}{|c|c|c|c|c|}
\hline Flagyl-USP & Flagyl-Mex & Colpofilin & Flagenase & Metral \\
\hline 0.50 & 1.07 & 2.20 & 4.74 & 3.33 \\
\hline 1.41 & 1.78 & 5.54 & 7.62 & 5.81 \\
\hline 2.54 & 3.56 & 7.78 & 11.47 & 12.10 \\
\hline 6.07 & 7.87 & 9.77 & 13.30 & 17.09 \\
\hline 10.28 & - & 18.11 & 24.19 & 27.08 \\
\hline
\end{tabular}

USP: United States Pharmacopeia.

\section{RESULTS}

\section{Assay and Disintegration Tests}

The assay and disintegration results are presented in Table 2. All tested drug products fell within the acceptance criteria of $90.0-110.0 \%$ of drug content (26). Among all the tested products, Flagyl-Mexico took the longest to disintegrate (around 18 minutes), followed by Metral, Flagenase, Flagyl-USP, and finally, Colpofilin.

Table 2. Drug Content and Disintegration Time of Different Commercially Available Metronidazole Immediate Release Tablets

\begin{tabular}{|c|c|c|c|c|}
\hline \multirow{2}{*}{ Product } & \multicolumn{2}{|c|}{ Assay } & \multicolumn{2}{c|}{ Disintegration } \\
\cline { 2 - 5 } & $\%$ & \multicolumn{1}{|c|}{ SD } & Time (min) & SD \\
\hline Flagyl-USP & 104.88 & 7.61 & 5.32 & 0.43 \\
\hline Flagyl-Mexico & 103.98 & 31.48 & 18.27 & 0.58 \\
\hline Flagenase & 104.68 & 7.56 & 10.16 & 0.07 \\
\hline Colpofilin & 102.02 & 62.61 & 0.60 & 0.22 \\
\hline Metral & 98.02 & 29.89 & 13.32 & 0.78 \\
\hline
\end{tabular}

USP: United States Pharmacopeia; SD, standard deviation. 


\section{Dissolution Tests and Data Analysis}

The dissolution results in the monophasic setup (900 $\mathrm{mL}$ ) are presented in Figure 1, including both SIF and low buffer capacity phosphate buffer $(5 \mathrm{mM})$. All tested products presented a similar performance in both buffer systems, as seen in Figure 1 and evidenced by the $f_{2}$ test results (Flagyl-USP: 49 [border line]; Flagyl-Mexico: 67; Flagenase: 54, Colpofilin: 82, Metral: 82). Since metronidazole is a highly soluble drug, its dissolution in a medium with lower buffer capacity is not expected to differ much from a highly concentrated buffer using a volume of $900 \mathrm{~mL}$ (Fig. 1) (14).

Except for Flagenase and Flagyl-USP, the dissolution rate followed the disintegration time, i.e., the faster the disintegration, the higher the release rate, even though all formulations were immediate-release dosage forms. Table 3 presents the statistical analysis results for the comparison between the different metronidazole products. As observed by Löbenberg et al., none of the tested metronidazole products were in vitro equivalent to the CPP or to other manufacturers in both monophasic dissolution experiments, with one exception of FlagylUSP and Metral in low buffer capacity $\left(f_{2}=73\right)(13)$.

Table 3. In Vitro Performance Comparison Between Metronidazole Products (similarity factor, $f_{2}$ )

\begin{tabular}{|c|c|c|c|c|}
\hline \multicolumn{5}{|c|}{ USP SIF } \\
\hline & Flagyl-USP & Flagyl-Mexico & Flagenase & Colpofilin \\
\hline Flagyl-USP & NA & - & - & - \\
\hline Flagyl-Mexico & 24 & NA & - & - \\
\hline Flagenase & 37 & 16 & NA & - \\
\hline Colpofilin & 23 & 10 & 32 & NA \\
\hline Metral & 48 & 30 & 32 & 19 \\
\hline & \multicolumn{5}{|c|}{ Low buffer capacity } & \\
\hline Flagyl-USP & NA & - & - & - \\
\hline Flagyl-Mexico & 28 & NA & - & - \\
\hline Flagenase & 41 & 20 & NA & - \\
\hline Colpofilin & 18 & 09 & 27 & NA \\
\hline Metral & 73 & 29 & 41 & 18 \\
\hline
\end{tabular}

USP: United States Pharmacopeia; SIF:simulated intestinal fluid; NA: not applicable; -: repeated.

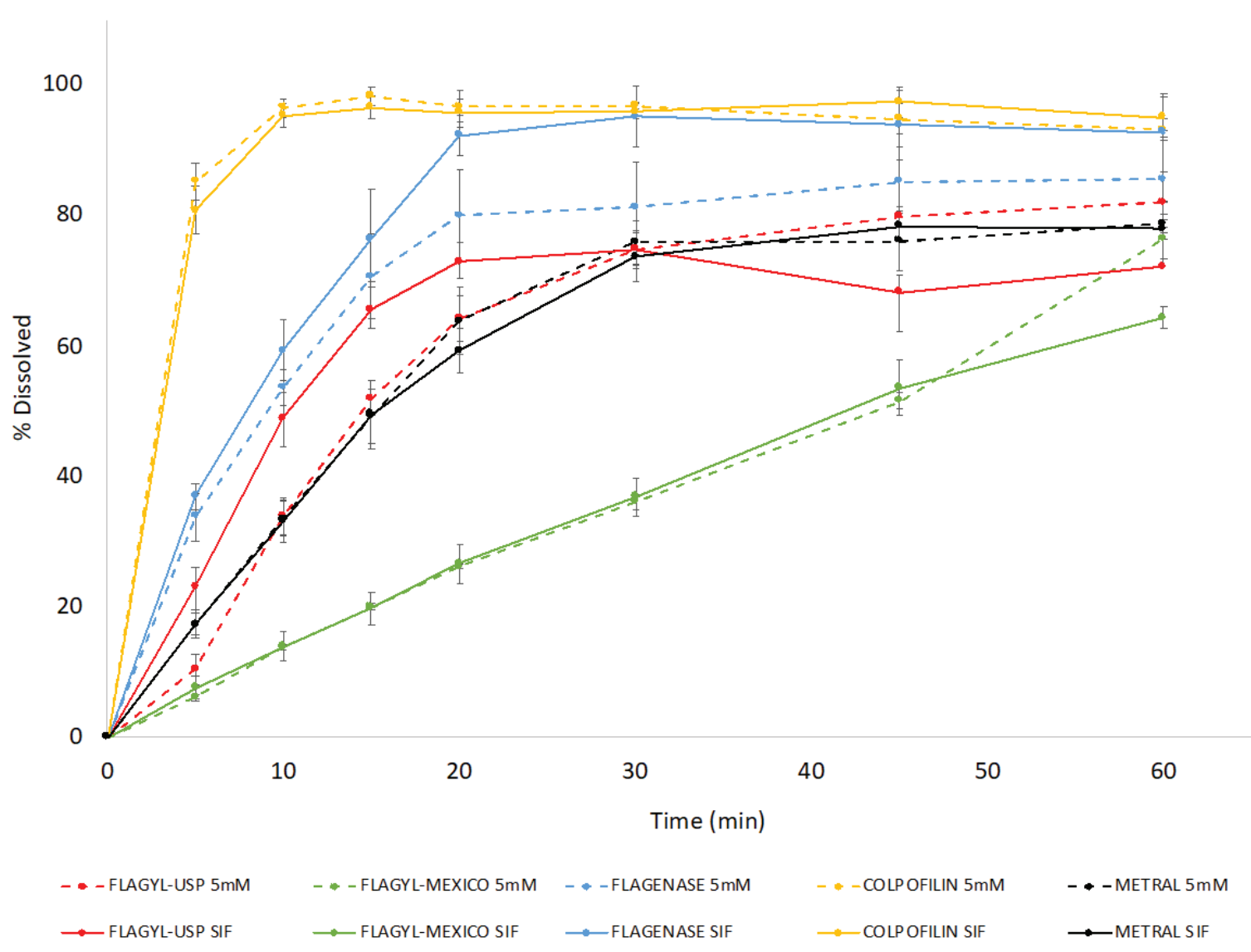

Figure 1. Dissolution profiles of metronidazole formulations in SIF (solid lines) and low buffer capacity phosphate buffer (5 mM - dashed lines). USP: United States Pharmacopeia; SIF: simulated intestinal fluid. 
The biphasic dissolution test results are presented in Figure 2. A similar pattern to the monophasic dissolution test was observed in the aqueous phase, i.e., Colpofilin having the highest dissolution rate, followed by Flagenase, Flagyl-USP, and Metral in the middle, and finally, FlagylMexico. Similarly, the partition profile followed the dissolution trend in aqueous phase and Metral was an exception.

Figure 3 shows the correlation of drug partitioned to the organic phase between the CPP and the generic drug products after disintegration. Notably, a good correlation was obtained in all cases $\left(R^{2} \geq 0.95\right)$, which could indicate similar in vivo performance. Further clinical studies would be needed to confirm interchangeability of these products.

\section{DISCUSSION}

Metronidazole is classified as a BCS class I drug (high solubility and high permeability). According to the current regulatory guidances, an API is highly soluble if its dose/ solubility ratio is $250 \mathrm{~mL}$ or less at the $\mathrm{pH}$ range of $1.0-6.8$ (or 7.5) at $37^{\circ} \mathrm{C}$ (4). For all definitions of dose (highest dose strength, highest dose recommended by $\mathrm{WHO}$, and the highest single dose administered), the ratio is below the 250-mL limit. The reported solubility of metronidazole in different aqueous medium at $37^{\circ} \mathrm{C}$ is $30.6 \mathrm{mg} / \mathrm{mL}(\mathrm{pH}$ 1.0); $14.1 \mathrm{mg} / \mathrm{mL}$ (pH 3.0); $12.8 \mathrm{mg} / \mathrm{mL}$ (pH 5.0); $11.6 \mathrm{mg} /$ $\mathrm{mL}(\mathrm{pH}$ 7.0). The reported log $\mathrm{P}$ value for metronidazole is 0.75 for n-octanol/water (27).

When applying the BCS-based biowaiver approach to immediate-release dosage forms containing highly soluble drugs, in vitro dissolution tests are to be conducted in at least three pharmacopeial buffer systems of $\mathrm{pH} 1.2$ (SGF), pH 4.5 (acetate buffer), and pH 6.8 (SIF). In these media, Löbenberg et al. reported that none of the tested metronidazole products were in vitro equivalent to the CPP (13).

According to the USP definition, immediate release is "a term for a dosage form in which no deliberate effort has been made to modify the drug substance release rate" (28). This definition lacks any mechanistic information, as pointed out by Uebbing et al. (28). A mechanistic understanding of the drug release is of primary importance because in cases in which the drug release is controlled by the API properties, disintegration is the

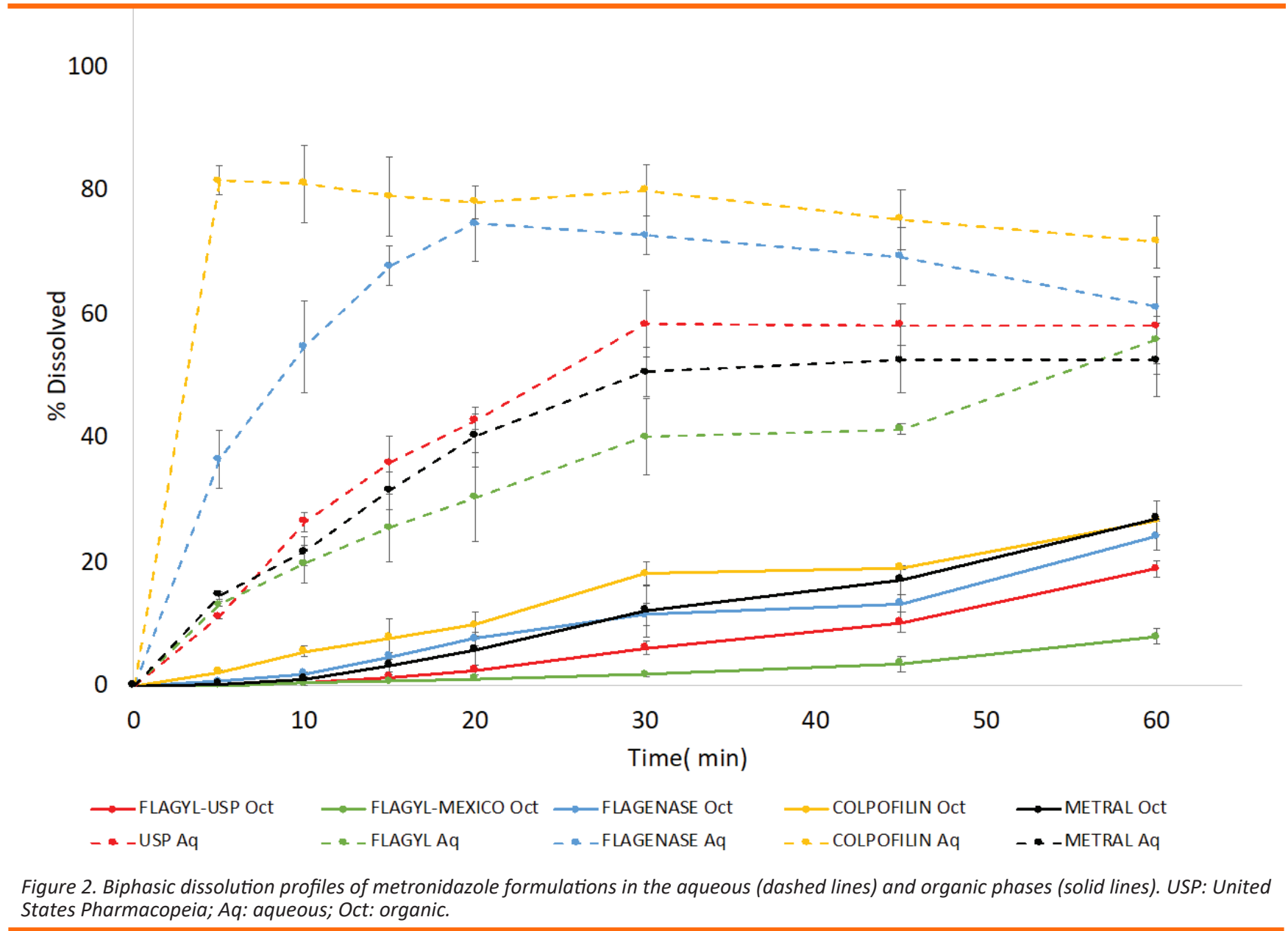




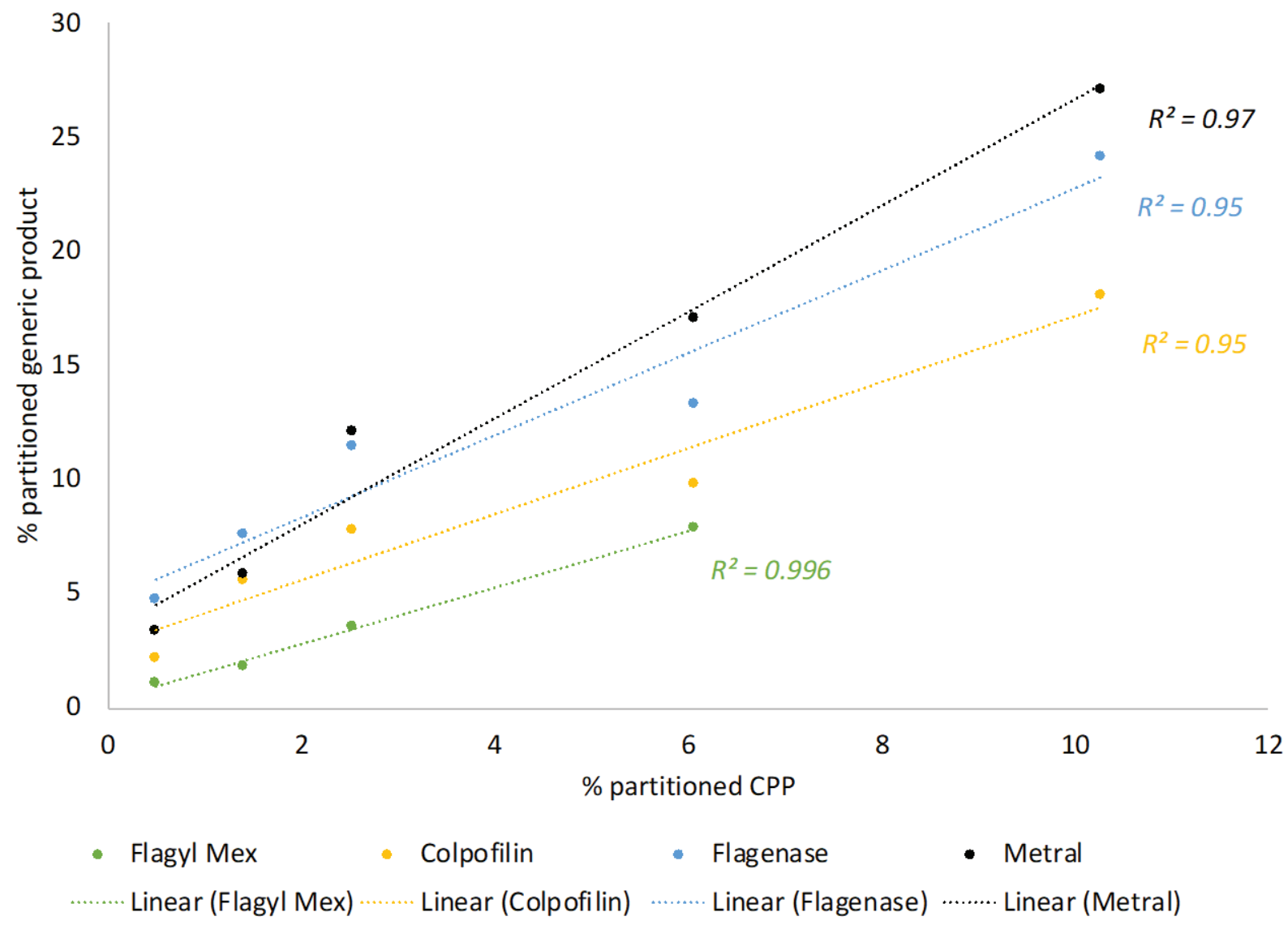

Figure 3. Correlation between the comparator pharmaceutical product (CPP) and generic products of the amount partitioned to the organic phase. Only data after complete tablet disintegration were used; Mex: Mexico

most important attribute of the dosage form. Hence, it could be used as a surrogate test for dissolution testing where the immediate-release formulation (containing a highly soluble drug) presents a fast disintegration time, such as Colpofilin ( $36 \mathrm{sec}$ ). As shown in Table 2 and Figure 1 , the disintegration time and release rate were quite different between the formulations. In this case, even though they were all identified/labelled as immediaterelease products, dissolution seems to be controlled by the formulation rather than the API itself, except for Colpofilin (28).

The similar performance of Colpofilin in both media indicates that buffer capacity does not affect the API's dissolution, since metronidazole is freely soluble in aqueous medium. Additionally, the other products' performances were not affected by buffer capacity. These results clearly indicate that aqueous dissolution is overdiscriminating.

With this in mind, using a more physiologically relevant dissolution system than compendial methods could be an additional in vitro approach to try before rendering the formulations inequivalent based solely on the compendial methods. In this study we used a biphasic dissolution system as a physiological relevant test, applying $5 \mathrm{mM}$ phosphate buffer (low buffer capacity) as the aqueous phase and n-octanol as the organic phase (14). The free drug concentration in the aqueous phase dictates the amount of drug that partitions to the organic phase, which acts as an absorptive compartment, mimicking the in vivo dissolution-absorption process in the intestinal lumen $(14,15,29)$. Since the drug is freely soluble in aqueous medium, the lipid dissolution could be the ratedetermining step for in vivo performance.

The stomach residence time value for half emptying under a fasted state, as reported in the literature, ranges from 11.5 to 17 minutes (30). The longest disintegration time among the products was 18 minutes for FlagylMexico. This means that, for all products, most likely the disintegration will take place within the stomach and metronidazole will be released out of the formulation and dissolved by the time it gets to the intestines. Hence, in the intestinal lumen, absorption would be the most important aspect. 
In the biphasic test, the organic phase profiles relate to the in vivo absorption. In the same way that only solubilized drug can be absorbed in vivo, only dissolved API molecules can partition to the organic phase. Disintegration and dissolution may occur simultaneously with the release of a drug from the dosage form. The drug particles that are on the surface of the tablet can be readily dissolved; however, disintegration is of utmost importance to completely release the drug. Accordingly, as seen in Figure 2 , even though drug dissolution and further partitioning can take place before complete disintegration of the dosage form (disintegration times presented in Table 2), the dissolution rate seems to follow disintegration time. Hence, it is most meaningful to correlate the partition profiles for each formulation after disintegration is complete (Fig. 3). When taking this mechanistic approach, good correlation was obtained between the CPP and the other formulations, which could be an indicator of similar in vivo performance. However, to assess the therapeutic equivalence among these products, an in vivo BE study should be conducted to corroborate the in vitro similarity in terms of partitioning profile.

There are many studies reporting the application of biphasic dissolution for poorly soluble drugs in various dosage forms (e.g., capsule, tablet, solution, suspension, etc.) with higher discriminating capacity, as well as better in vitro-in vivo relationships (20). However, there are not many studies using this model applied to BCS class I drugs, due to the good solubility of such drugs and the assumption that pharmacopeial methods might be sufficient to discriminate between biopharmaceutical properties. Hence, the results of the presentstudy broaden the application of biphasic dissolution demonstrating that it is a plausible alternative for highly soluble drugs.

In the case of poorly soluble drugs, the organic phase acts as an additional sink as the drug is removed from the aqueous phase, preventing aqueous saturation. Since metronidazole is a highly soluble drug, the drug removal from the aqueous phase had low-to-neglectable effects on the aqueous dissolution. It was then further evidenced that for most products tested, the formulation was the factor of pivotal importance in controlling the dissolution rate. After disintegration, metronidazole quickly dissolves, and permeability becomes the most crucial aspect. A mechanistic formulation evaluation and an understanding of factors controlling dissolution and in vivo processes highlights that biphasic systems may be used for highly soluble drugs. Our results delineate the potential in vitro equivalence between different drug manufacturers and the CPP for metronidazole, indicating that the compendial methods utilized previously might have been overdiscriminating and can be further optimized.

\section{CONCLUSION}

None of the tested metronidazole products demonstrated in vitro equivalence to the CPP in the monophasic dissolution media, i.e., SIF and physiological buffer capacity. Hence, the monophasic aqueous systems seem to be overdiscriminating. On the other hand, correlation of the organic phase in the biphasic system presented a similar partitioning pattern for all tested drug products and the CPP, which could indicate in vitro equivalence. The application of biphasic dissolution to highly soluble drugs and formulations has beneficial attributes to estimate the in vitro behavior and performance. Further in vitro studies with other products are needed to confirm and refine these findings. An in vivo BE study is needed to assess the therapeutic equivalence among these products.

\section{ACKNOWLEDGMENT}

Daniela Amaral Silva is supported by the Alberta Innovates Graduate Student Scholarship. Opinions, interpretations, conclusion, and recommendations are those of the author and not necessarily endorsed by the funding agency.

The authors would like to thank Sotax AG (Switzerland) for kindly providing the mini paddles used in this study.

\section{CONFLICT OF INTEREST}

The authors disclosed no conflicts of interest related to this article.

\section{REFERENCES}

1. Amidon, G. L.; Lennernäs, H.; Shah, V. P.; Crison, J. R. A theoretical basis for a biopharmaceutic drug classification: the correlation of in vitro drug product dissolution and in vivo bioavailability. Pharm. Res. 1995, 12, 413-420. DOI: 10.1023/A:1016212804288.

2. Gupta, E.; Barends, D. M.; Yamashita, E.; Lentz, K. A.; Harmsze, A. M.; Shah, V. P.; Dressman, J. B.; Lipper, R. A. Review of global regulations concerning biowaivers for immediate release solid oral dosage forms. Eur. J. Pharm. Sci. 2006, 29, 315-324. DOI: 10.1016/j.ejps.2006.05.001.

3. Löbenberg, R.; Amidon, G. L. Modern Bioavailability, Bioequivalence and Biopharmaceutics Classification System. New Scientific Approaches to International Regulatory Standards. Eur. J. Pharm. Biopharm. 2000, 50, 3-12. DOI: 10.1016/S0939-6411(00)00091-6.

4. Waiver of In Vivo Bioavailability and Bioequivalence Studies for Immediate-Release Solid Oral Dosage Forms Based on a Biopharmaceutics Classification System; Guidance for Industry; U.S. Department of Health and Human 
Services, Food and Drug Administration, Center for Drug Evaluation and Research (CDER), U.S. Government Office: Washington, DC; 2000.

5. WHO Expert Committee on Specifications for Pharmaceutical Preparations. Multisource (generic) pharmaceutical products: guidelines on registration requirements to establish interchangeability; WHO Technical Report Series, No. 937, Annex 7; World Health Organization: Geneva, 2006.

6. Note for Guidance on the Investigation of Bioavailability and Bioequivalence; CPMP/EWP/QWP/1401/98; Committee for Proprietary Medicinal Products (CPMP), European Medicines Agency: London, 2001.

7. Hofsäss, M. A.; Dressman, J. B. The discriminatory power of the BCS-Based biowaiver: a retrospective with focus on essential medicines. J. Pharm. Sci. 2019, 108, 2824-2837. DOI: 10.1016/j.xphs.2019.04.030.

8. Bou-Chacra, N.; Melo, K. J. C.; Morales, I. A. C.; Stippler, E. S.; Kesisoglou, F.; Yazdanian, M.; Löbenberg, R. Evolution of choice of solubility and dissolution media after two decades of biopharmaceutical classification system. AAPS J. 2017, 19, 989-1001. DOI: 10.1208/s12248-017-0085-5.

9. Guideline on the Investigation of Bioequivalence; CPMP/ EWP/QWP/1401/98 Rev. 1; Committee for Medicinal Products for Human Use (CHMP), European Medicines Agency: London, 2010.

10. WHO Expert Committee on Specifications for Pharmaceutical Preparations. Multisource (generic) pharmaceutical products: guidelines on registration requirements to establish interchangeability; WHO Technical Report Series, No. 1003, Annex 6; World Health Organization: Geneva, 2017.

11. Waiver of In Vivo Bioavailability and Bioequivalence Studies for Immediate-Release Solid Oral Dosage Forms Based on a Biopharmaceutics Classification System; Guidance for Industry; U.S. Department of Health and Human Services, Food and Drug Administration, Center for Drug Evaluation and Research (CDER), U.S. Government Office: Silver Spring, MD; 2017.

12. ICH M9 Guidelines on Biopharmaceutics Classifcation System-Based Biowaivers; EMA/CHMP/ICH/493213/2018; Committee for Medicinal Products for Human Use (CHMP), European Medicines Agency: London, 2020.

13. Löbenberg, R.; Chacra, N. B.; Stippler, E. S.; Shah, V. P.; DeStefano, A. J.; Hauck, W. W.; Williams, R. L. Toward global standards for comparator pharmaceutical products: case studies of amoxicillin, metronidazole, and zidovudine in the Americas. AAPS J. 2012, 14, 462-472. DOI: 10.1208/ s12248-012-9350-9.

14. Amaral Silva, D. A.; Al-Gousous, J.; Davies, N. M.; Chacra, N. B.; Webster, G. K.; Lipka, E.; Amidon, G. L.; Löbenberg, R. Biphasic dissolution as an exploratory method during early drug product development. Pharmaceutics 2020, 12, 420. DOI: 10.3390/pharmaceutics12050420.

15. Mudie, D. M.; Shi, Y.; Ping, H.; Gao, P.; Amidon, G. L.; Amidon, G. E. Mechanistic analysis of solute transport in an in vitro physiological two-phase dissolution apparatus. Biopharm. Drug Dispos. 2012, 33, 378-402. DOI: 10.1002/ bdd.1803.

16. Phillips, D. J.; Pygall, S. R.; Cooper, V. B.; Mann, J. C. Overcoming sink limitations in dissolution testing: a review of traditional methods and the potential utility of biphasic systems. J. Pharm. Pharmacol. 2012, 64, 1549-1559. DOI: 10.1111/j.2042-7158.2012.01523.x.

17. Vangani, S.; Li, X.; Zhou, P.; Del-Barrio, M.-A.; Chiu, R.; Cauchon, N.; Gao, P.; Medina, C.; Jasti, B. Dissolution of poorly water-soluble drugs in biphasic media using USP 4 and fiber optic system. Clin. Res. Regul. Aff. 2009, 26, 8-19. DOI: 10.1080/10601330902905887.

18. AlDurdunji, A.; AlKhatib, H. S.;Al-Ghazawi, M. Development of a biphasic dissolution test for Deferasirox dispersible tablets and its application in establishing an in vitro-in vivo correlation. Eur. J. Pharm. Biopharm. 2016, 102, 9-18. DOI: 10.1016/j.ejpb.2016.02.006.

19. Pestieau, A.; Evrard, B. In vitro biphasic dissolution tests and their suitability for establishing in vitro-in vivo correlations: a historical review. Eur. J. Pharm. Sci. 2017, 102, 203-219. DOI: 10.1016/j.ejps.2017.03.019.

20. Deng, J.; Staufenbiel, S.; Hao, S.; Wang, B.; Dashevskiy, A.; Bodmeier, R. Development of a discriminative biphasic in vitro dissolution test and correlation with in vivo pharmacokinetic studies for differently formulated racecadotril granules. J. Control. Release 2017, 255, 202209. DOI: 10.1016/j.jconrel.2017.04.034.

21. Xu, H.; Shi, Y.; Vela, S.; Marroum, P.; Gao, P. Developing quantitative in vitro-in vivo correlation for fenofibrate immediate-release formulations with the biphasic dissolution-partition test method. J. Pharm. Sci. 2018, 107, 476-487. DOI: 10.1016/j.xphs.2017.06.018.

22. Shi, Y.; Gao, P.; Gong, Y.; Ping, H. Application of a biphasic test for characterization of in vitro drug release of immediate release formulations of celecoxib and its relevance to in vivo absorption. Mol. Pharm. 2010, 7, 1458-1465. DOI: 10.1021/mp100114a.

23. Deng, J.; Staufenbiel, S.; Bodmeier, R. Evaluation of a biphasic in vitro dissolution test for estimating the bioavailability of carbamazepine polymorphic forms. Eur. J. Pharm. Sci. 2017, 105, 64-70. DOI: 10.1016/j. ejps.2017.05.013.

24. <701> Disintegration. In The United States Pharmacopeia and National Formulary USP 43-NF 38 2S; The United States Pharmacopeial Convention, Inc.: Rockville, MD, 2020.

25. Mudie, D. M.; Murray, K.; Hoad, C. L.; Pritchard, S. E.; Garnett, 
M. C.; Amidon, G. L.; Gowland, P. A.; Spiller, R. C.; Amidon, G. E.; Marciani, L. Quantification of gastrointestinal liquid volumes and distribution following a $240 \mathrm{~mL}$ dose of water in the fasted state. Mol. Pharm. 2014, 11, 3039-3047. DOI: 10.1021/mp500210c.

26. $<2>$ Oral drug products-product quality tests. In The United States Pharmacopeia and National Formulary USP 43-NF 38 2S; The United States Pharmacopeial Convention, Inc.: Rockville, MD, 2020.

27. Rediguieri, C. F.; Porta, V.; G. Nunes, D. S.; Nunes, T. M.; Junginger, H. E.; Kopp, S.; Midha, K. K.; Shah, V. P.; Stavchansky, S.; Dressman, J. B.; Barends, D. M. Biowaiver monographs for immediate release solid oral dosage forms: metronidazole. J. Pharm. Sci. 2011, 100, 1618-1627. DOI: 10.1002/jps.22409.

28. Uebbing, L.; Klumpp, L.; Webster, G. K.; Löbenberg, R. Justification of disintegration testing beyond current fda criteria using in vitro and in silico models. Drug Des. Devel. Ther. 2017, 11, 1163-1174. DOI: 10.2147/DDDT.S131213.

29. Hens, B.; Tsume, Y.; Bermejo, M.; Paixao, P.; Koenigsknecht, M. J.; Baker, J. R.; Hasler, W. L.; Lionberger, R.; Fan, J.; Dickens, J.; Shedden, K.; Wen, B.; Wysocki, J.; Loebenberg, R.; Lee, A.; Frances, A.; Amidon, G.; Yu, A.; Benninghoff, G.; Salehi, N.; Talattof, A.; Sun, D.; Amidon, G. L. Low buffer capacity and alternating motility along the human gastrointestinal tract: implications for in vivo dissolution and absorption of ionizable drugs. Mol. Pharm. 2017, 14, 4281-4294. DOI: 10.1021/acs.molpharmaceut.7b00426.

30. Steingoetter, A.; Fox, M.; Treier, R.; Weishaupt, D.; Marincek, B.; Boesiger, P.; Fried, M.; Schwizer, W. Effects of posture on the physiology of gastric emptying: a magnetic resonance imaging study. Scand. J. Gastroenterol. 2006, 41, 1155-1164. DOI: 10.1080/00365520600610451. 